\title{
MicroRNA-21 as a biomarker and potential therapeutic target in pancreatic ductal adenocarcinoma
}

Authors: Agata Kolasa, Zuzanna Gwóźdź, Alicja Groszek, Joanna Kobak, Norbert Nowak (mentor), Andrzej Dąbrowski (mentor)

2nd Department Of General And Gastrointestinal Surgery And Surgical Oncology Of Alimentary Tract, Medical University Of Lublin, Lublin, Poland

DOI: https://doi.org/10.26800/LV-142-supp5-56

\section{Introduction:}

Pancreatic ductal adenocarcinoma (PDAC) has the worst prognosis among all cancers due to latestage diagnosis, limited detection and treatment methods. MiRNAs are post-transcriptional regulators, recently reported to have numerous functions, including modulation of cellular differentiation, proliferation, and apoptosis, Several tumor suppressors, such as PTEN, TPM1, and PDCD4 were identified as miR-21 targets. MiR-21 is stable in both tissues and plasma, disease-specific and therefore it appears to be a promising biomarker for benign and malignant diseases of pancreas. Current evidence suggests that miR-21 overexpression is associated with shorter overall survival, higher rate of liver and regional lymph nodes metastases.

\section{Aim of study:}

The purpose of the study is to review the current literature on the evaluation of miR-21 as a prognostic biomarker in PDAC, as well as influence of miR-21 on pancreatic cancer cells.

\section{Materials and Methods:}

Review of English language articles from PubMed addressing the link between miR-21 and PDAC.

\section{Results:}

In the analysed articles, increased miR-21 is associated with significant decrease of overall and progression-free survival in patients with PDAC. In addition miR-21 upregulation is linked with poor tumor differentiation and lymph nodes metastasis regardless of sample source, either tissue or circulation samples. The miR-21 has been found to be elevated very early in pancreatic neoplasia. Interestingly, inhibition of miR-21 decreased chemoresistance for gemcitabine of pancreatic cancer cells.

\section{Conclusion:}

According to the latest literature, miR-21 contributed to proliferation, invasion, and chemoresistance of pancreatic cancer cells. In conclusion, miR-21 may serve as a useful prognostic biomarker and potential target for pancreatic cancer therapy.

Keywords: biomarker, miRNA, pancreatic cancer, prognosis, 